\title{
DISEÑO DE UN SISTEMA DE EVALUACIÓN BASADO EN LAS NORMAS HACCP DIRECCIONADO A POTENCIAR LA INDUSTRIA ALIMENTARIA ECUATORIANA-SECTOR MOLINERO
}

\author{
Manuel Alejandro Jaramillo Recalde ${ }^{1}$, Lorena Del Pilar Ramos Paucar ${ }^{2}$, Silvia Melinda Oyaque Mora ${ }^{3}$. \\ alejandroj09@gmail.com ${ }^{1}$, lore_mos22@hotmail.com², sm.oyaque@uta.edu.ec ${ }^{3}$ \\ Universidad Técnica de Ambato ${ }^{1}{ }^{3}$, Consejo Nacional Electoral ${ }^{2}$ \\ Ambato - Ecuador
}

Recibido (13/07/20), Aceptado (28/07/20)

\begin{abstract}
Resumen: Los peligros y puntos críticos de control HACCP se convierte en un requisito fundamental para la apertura de nuevos mercados y oportunidades de exportación de productos alimenticios, se crea directrices para la aplicación de este sistema basado en literatura de organizaciones académicas y de control sanitario, con miras al cumplimiento de disposiciones gubernamentales de buenas prácticas de manufactura que garantizan la producción de alimentos de carácter inocuo. Por ello, el presente estudio pretende determinar si el diseño de un sistema de evaluación basado en las normas HACCP potenciaría la industria alimentaria ecuatoriana. Se utiliza el método cualitativo de Investigación - Acción en conjunto con el propuesto por Food and Drug Administration FDA apartado Principios de HACCP y Directrices de Aplicación para la implementación del sistema HACCP. Se concluye que en el Ecuador en su proceso de cambio de matriz productiva necesita incrementar el número de industrias alimenticias que posean sistemas de gestión de calidad e inocuidad, para ello sirve este trabajo como guía o pauta para la implementación del sistema de Evaluación basado en las HACCP..
\end{abstract}

Palabras Clave: Punto crítico de control, HACCP, Sistema de Evaluación, Industria Alimentaria.

\section{DESIGN OF AN EVALUATION SYSTEM BASED ON HACCP STANDARDS AIMED AT STRENGTHENING THE ECUADORIAN FOOD INDUSTRY-MILLER SECTOR}

\begin{abstract}
The dangers and critical control points HACCP becomes a fundamental requirement for the opening of new markets and opportunities for the export of food products, guidelines are created for the application of this system based on literature from academic and health control organizations, with a view to to the fulfillment of governmental dispositions of good manufacturing practices that guarantee the production of innocuous food. Therefore, this study aims to determine whether the design of an evaluation system based on HACCP standards would enhance the Ecuadorian food industry. The qualitative ResearchAction method is used in conjunction with the one proposed by the Food and Drug Administration FDA, section HACCP Principles. and Application Guidelines for the implementation of the HACCP system. It is concluded that in Ecuador, in its process of changing the production matrix, it needs to increase the number of food industries that have quality and safety management systems, for this purpose this work serves as a guide or guideline for the implementation of the Evaluation system based on the HACCP.
\end{abstract}

Keywords: Critical control point, HACCP, Evaluation System, Food. 


\section{I.INTRODUCCIÓN}

El cambio de la matriz productiva en el Ecuador provoca que para las empresas industriales ecuatorianas se vea más complicado sus procesos de expansión a nivel internacional y su desarrollo de mercados acorde a requerimientos normativos de sistemas de gestión de calidad e inocuidad [1]. Por tal motivo, la presente investigación presenta el desarrollo del sistema de evaluación basado en el análisis de peligros y puntos críticos de control HACCP en el procesamiento de harina de trigo.

El acrónimo HACCP significa Análisis de Peligros y Puntos Críticos de Control, es un sistema de seguridad alimentaria basada en la prevención. Los sistemas HACCP están diseñados para prevenir la aparición de posibles problemas de seguridad alimentaria. Esto se logra mediante la evaluación de los riesgos inherentes atribuibles a un producto o un proceso y luego determinar las medidas necesarias que controlarán los riesgos identificados [2]. El objetivo del sistema HACCP es identificar los peligros relacionados con la seguridad del consumidor que puedan ocurrir en la cadena alimentaria, estableciendo los procesos de control para garantizar la inocuidad del producto, en base a lo establecido el presente estudio pretende determinar si el diseño de un sistema de evaluación basado en las normas HACCP potenciaría la industria alimentaria ecuatoriana.

Se utiliza un método de investigación cualitativa Investigación-Acción, en conjunto con la guía y principios para implementación de sistemas HACCP de Food and Drug Administration. Se conforma el equipo HAC$\mathrm{CP}$, se describe el alimento, distribución, uso previsto y consumidores; también se desarrolla un diagrama de flujo para describir y verificar el proceso. Se realiza un análisis de peligros determinando el punto crítico de control (PCC): Tamizado MKZF y se establece límites críticos en su operación.

Se elabora procedimientos de monitoreo, acciones correctivas e instrucciones de verificación para asegurar la inocuidad del producto. La industria molinera de trigo del país no cuenta con directrices ni sistemas de gestión de calidad e inocuidad alimentaria basados en el sistema de análisis de peligros y puntos críticos de control (HACCP) y se torna indispensable garantizar la satisfacción del cliente y proteger la salud del mercado ecuatoriano de alimentos, transformando la Industria no sólo con producción de materias primas sino haciéndola competitiva internacionalmente con valor agregado [3].

\section{II.DESARROLLO}

\section{A.Sistema HACCP}

El sistema HACCP es compatible con otros siste- mas de control de calidad. Esto significa que inocuidad, calidad y productividad pueden abordarse en conjunto, resultando en beneficios para los consumidores, más ganancias para las empresas y mejores relaciones entre todas las partes que participan, en función del objetivo común de garantizar la inocuidad y la calidad de los alimentos. Todo esto se expresa en evidente beneficio para la salud y para la economía de los países [4]; [5].

El sistema HACCP se basa en la existencia de sistemas de gestión de la calidad sólidamente implantados, como las buenas prácticas de fabricación (BPF), las buenas prácticas de higiene (BPH), las buenas prácticas agrícolas (BPA) y las buenas prácticas de almacenamiento (BPAL) [6]. La Norma Chilena NCh 2861-2011, sugiere antes de aplicar el sistema HACCP, la empresa debe tener implementado los programas de prerrequisitos, los cuales deben estar escritos y actualizados [7].

Se consideran prerrequisitos para el funcionamiento de un sistema HACCP, las Buenas Prácticas de Manufactura (BPM); las Buenas Prácticas de Fabricación (BPF); las Buenas Prácticas de Elaboración (BPE); y las Buenas Prácticas de Producción (BPP), los Procedimientos Operacionales Estandarizados (POE) y los Procedimientos Operacionales Estandarizados de Sanitización (POES), puesto que en éstos se fundamentan, en gran parte, las medidas de control sugeridas en el plan [8].

La producción de productos alimenticios seguros requiere que el sistema HACCP sea construido bajo una sólida base de programas de requisitos previos cada segmento de la industria alimentaria debe proporcionar las condiciones necesarias para proteger los alimentos mientras está bajo su control [9]. Este tradicionalmente se ha logrado mediante la aplicación de GMP (Good Manufacturing Practices). Estas condiciones y prácticas están consideradas como requisito previo para el desarrollo y ejecución de los planes de HACCP eficaz.

Los programas previos proporcionan las condiciones operativas y ambientales básicas que son necesarias para la producción de alimentos seguros y saludables. Muchas de las condiciones y prácticas están especificadas en los reglamentos y directrices (por ejemplo, GMP y Código Alimentario) federales, estatales y locales. Los Principios Generales del Codex Alimentarius de Higiene de los Alimentos describen las condiciones y prácticas básicas que se esperan para los alimentos destinados al comercio internacional.

\section{B.Reglamento de Buenas Prácticas de Manufactura para alimentos procesados}

En el Ecuador, El Reglamento de Buenas Prácticas de Manufactura para Alimentos Procesados fue expedi- 
do bajo Decreto Ejecutivo No. 3253 - 24 de Octubre de 2002. Las Enfermedades Transmitidas por Alimentos (ETA) han sido reconocidas como el problema de salud pública más extendido en el mundo actual, provocando una importante de la productividad y grandes pérdidas económicas que afectan a países, empresas, pequeños negocios familiares y a los consumidores [10].

El Sistema HACCP fue diseñado para controlar el proceso de producción, y se basa en principios y conceptos preventivos (CIGI, 2014). Todas las personas que participan del sector productivo de alimentos deben estar comprometidas en la implementación del sistema de evaluación, los principios HACCP y la elaboración del plan HACCP [4]. Establecer un programa de HAC$\mathrm{CP}$ permite enfatizar más en la prevención que en la detección, disminuye los costos, minimiza el riesgo de fabricar productos defectuosos, ofrece mayor confianza a la gerencia, fortalece la competitividad nacional e internacional, entre otras ventajas [11].

El comercio internacional de alimentos es regulado por la Organización Mundial del Comercio (OMC), garantizando que todas las relaciones económicas de alimentos sean controladas por normas, directrices y recomendaciones de la Comisión del Codex Alimentarius, de la Organización Mundial de Sanidad Animal (OIE) y de la Convención Internacional de Protección Fitosanitaria (IPPC o CIPF). Las normas y, directrices y otras recomendaciones del Codex se tornaron la base para la producción de alimentos inocuos y la protección del consumidor en el comercio internacional de alimentos [4]. La industria molinera del trigo en Ecuador, depende totalmente de la oferta extranjera de su principal insumo, ya que utiliza en el 99\% materia prima importada, es decir trigo que proviene en relación a su importancia de: Canadá, EEUU y Argentina [12].

Las Buenas Prácticas de Elaboración (BPE) y las Buenas Prácticas de Producción (BPP) son el instrumento para evaluar los peligros y establecer sistemas de control que se centren en la prevención, en lugar de basarse principalmente en el ensayo del producto final. Se debe tomar en consideración también: Peligro significativo Plan HACCP, Procedimientos Operacionales Estandarizados; POE; SOP, Procedimientos Operacionales Estandarizados de Sanitización; POES; SSOP, Punto Crítico de Control; PCC, Punto de Control; PC [7]; [13],

\section{III.METODOLOGÍA}

Se aplicó un proceso de investigación cualitativa, con un diseño de Investigación - Acción. La investigación-acción envuelve la transformación y mejora de una realidad (social, educativa, administrativa). De hecho, se construye una parte de problemas prácticos y vinculados con un ambiente o entorno, con la colaboración de los participantes en la detección de necesidades y la implementación de los resultados del estudio [14].

Para promover mercados de exportación y asegurar la inocuidad del producto, en este caso se utilizó la experiencia y conocimiento de sus colaboradores para formar un equipo multidisciplinario y lograr la implementación de un sistema que garantice que sus consumidores no sufran daños a la salud. Se utilizó este método cualitativo de Investigación - Acción en conjunto con el propuesto por Food and Drug Administration FDA en su apartado Principios de HACCP y Directrices de Aplicación para la implementación del sistema HACCP. Los cuales fueron: Formación del equipo HACCP, describir el alimento y su distribución, describir el uso previsto y los consumidores del alimento, desarrollar un diagrama de flujo que describe el proceso.

Se trabajó bajo los siguientes principios: a) Realizar un análisis de peligros; b) Principio; c) Determinar los puntos críticos de control (PCC); d) Establecer límites críticos; e) Establecer procedimientos de monitoreo; f) Establecer acciones correctivas; g) Establecer procedimientos de verificación y h) Establecer el mantenimiento de registros y procedimientos de documentación.

\section{IV.RESULTADOS}

El equipo HACCP deberá estar conformado por personal familiarizado con los productos elaborados, métodos, el flujo y el equipo de producción, así como con las instalaciones físicas. En conjunto el equipo de trabajo deberá a) Estar familiarizado con los principios del sistema HACCP; b) Poder anticipar y detectar los posibles riesgos y su grado de peligrosidad, así como cualquier problema en los productos y /o procesos; c) Tener conocimientos para llevar a cabo cambios, tanto en los procesos como en los productos que garanticen una mayor seguridad e integridad y d) Ser capaz de comunicar efectivamente los cambios requeridos al personal responsable de las operaciones.

Para la aplicación del Sistema de Evaluación se tomará en consideración un producto específico y será sometido a todo el proceso resultado de nuestra investigación como el trigo, debido a que es el grano obtenido de las variedades de la especie Triticum aestivum, pertenece al género Triticum, de la familia Gramínea. Se trabajó también con los componentes del trigo, especies de trigo origen y citogenética, especificaciones del producto elaborado, condiciones del transporte, uso esperado del producto y consumidores.

En las tablas siguientes se contempla los análisis realizados, para comprensión de las tablas se deter- 
minan las siguientes abreviaturas: PQ: Peligro químico; PB: Peligro biológico y PF: Peligro físico/ cuerpos extraños. Ahora para el punto crítico de control (PCC) se trabaja con los siguientes valores: G: Gravedad (1= Menor; $2=$ Moderado; $3=$ Serio y $4=$ Muy serio y $\mathrm{O}$ : Ocurrencia $(1=$ Remota; $2=$ Ocasional; $3=$ Probable y 4= Frecuente)

Para determinar los puntos críticos de control la in- formación relacionada se procedió a analizar todos los procesos en la cadena de producción, de los cuales se tomaron los que correspondían a riesgos significativos una vez determinados los riesgos significativos, para su interpretación se debe considerar PQ: Peligro químico; PB: Peligro biológico; PF: Peligro físico/ cuerpos extraños y PCC: Punto crítico de control.

Tabla I. Determinación de punto crítico de control - Tamizado de control MKZF

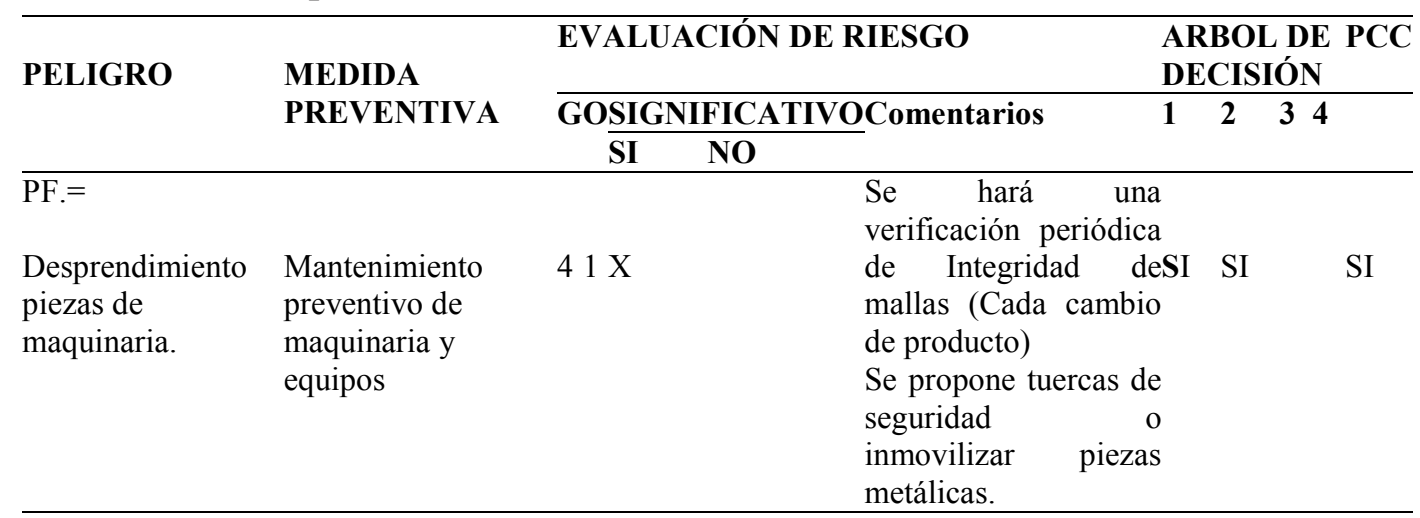

Fuente. Elaborador por los autores

Para establecer límites críticos se basa principalmente en los estudios científicos realizados por Food and Drug Administration en su apartado CPG Sec.
555.425 Foods, Adulteration Involving hard or Sharp Foreign Objects.

Tabla II. Límites críticos para el punto crítico de control - Tamizado de control MKZF

\begin{tabular}{|c|c|c|c|}
\hline PCC & Producto & Peligro & Límite crítico \\
\hline \multirow[t]{2}{*}{$\begin{array}{l}\text { Tamizado de control } \\
\text { turbotamizadora MKZF }\end{array}$} & Harinas & $\begin{array}{l}\text { Partículas extrañas al } \\
\text { producto cuyo tamaño } \\
\text { pueda afectar a la }\end{array}$ & $\begin{array}{l}\text { Presencia de partículas } \\
\text { extrañas al producto } \\
\text { mayores a } 3 \mathrm{~mm}\end{array}$ \\
\hline & Harinas Integrales & $\begin{array}{l}\text { integridad o la salud } \\
\text { humana }\end{array}$ & $\begin{array}{l}\text { Presencia de partículas } \\
\text { extrañas al producto } \\
\text { mayores a } 6 \mathrm{~mm}\end{array}$ \\
\hline
\end{tabular}

Fuente. Elaborador por los autores

Se establece el procedimiento de monitoreo del punto crítico de control, en donde, se realizó una revisión de la integridad de la malla que compone el Tamizado de control turbotamizadora MKZF, el monitoreo se efectuó de forma visual para identificar roturas de malla con un periodo de cada cuatro horas y/o cada cambio de lote de harina.

Tabla III. Monitoreo de punto crítico de control - Tamizado de control MKZF

\begin{tabular}{|c|c|c|c|c|c|c|}
\hline PCC & Producto & Límite crítico & Qué & Cómo & Cuando & Quién \\
\hline \multirow{2}{*}{$\begin{array}{l}\text { Tamizado de } \\
\text { control } \\
\text { turbotamizadora } \\
\text { MKZF }\end{array}$} & Harina & $\begin{array}{l}\text { Presencia de } \\
\text { partículas } \\
\text { mayores a } 3 \mathrm{~mm}\end{array}$ & \multirow[t]{2}{*}{$\begin{array}{l}\text { Revisión } \\
\text { integridad } \\
\text { de malla }\end{array}$} & \multirow[t]{2}{*}{ Visual } & \multirow{2}{*}{$\begin{array}{lr}\text { Cada } 4 \\
\text { horas } y / 0 \\
\text { cada } \\
\text { cambio de } \\
\text { lote de } \\
\text { harina }\end{array}$} & \multirow[t]{2}{*}{ Producción } \\
\hline & Harina Integral & $\begin{array}{l}\text { Presencia } \mathrm{de} \\
\text { partículas } \\
\text { mayores a } 6 \mathrm{~mm}\end{array}$ & & & & \\
\hline
\end{tabular}

Fuente. Elaborador por los autores 
Para establecer las acciones correctivas se recopiló información del Equipo HACCP y se determinó que al desviarse el proceso del límite crítico se debe realizar lo siguiente: Parar el proceso; se procederá a separar el producto; se comunicará al Supervisor de Producción o al personal de Control de Calidad; se realizará el cambio de malla de la Turbotamizadora MKZF; se reprocesará el producto; el equipo establecerá el respectivo análisis de causa.

Tabla IV. Establecimiento de acciones correctivas

\begin{tabular}{|c|c|c|c|c|}
\hline $\begin{array}{l}\text { PCC } \\
\text { Tamizado de } \\
\text { control } \\
\text { turbotamizadora } \\
\text { MKZF }\end{array}$ & Producto & Peligro & Límite crítico & $\begin{array}{l}\text { Acciones correctivas } \\
\text { En caso de ruptura de la malla se } \\
\text { tendrá que: } \\
\text { 1. Parar el proceso. } \\
\text { 2. Se procederá a separar el } \\
\text { producto. } \\
\text { 3. Se comunicará al Supervisor de } \\
\text { Producción o al personal de } \\
\text { Control de Calidad. } \\
\text { 4. Se realizará el cambio de malla. } \\
\text { 5. Se reprocesará el producto. } \\
\text { 6. El equipo establecerá el } \\
\text { respectivo análisis de causa. }\end{array}$ \\
\hline \multirow{9}{*}{$\begin{array}{l}\text { Tamizado de } \\
\text { control } \\
\text { turbotamizadora } \\
\text { MKZF }\end{array}$} & Harinas & \multirow{9}{*}{$\begin{array}{l}\text { Partículas } \\
\text { extrañas al } \\
\text { producto } \\
\text { cuyo } \\
\text { tamaño } \\
\text { pueda } \\
\text { afectar a la } \\
\text { integridad } \\
\text { o la salud } \\
\text { humana }\end{array}$} & $\begin{array}{lr}\text { Presencia } & \text { de } \\
\text { partículas } & \text { extrañas }\end{array}$ & \multirow{9}{*}{$\begin{array}{l}\text { En caso de ruptura de la malla se } \\
\text { tendrá que: } \\
\text { 1. Parar el proceso. } \\
\text { 2. Se procederá a separar el } \\
\text { producto. } \\
\text { 3. Se comunicará al Supervisor de } \\
\text { Producción o al personal de } \\
\text { Control de Calidad. } \\
\text { 4. Se realizará el cambio de malla. } \\
\text { 5. Se reprocesará el producto. } \\
\text { 6. El equipo establecerá el } \\
\text { respectivo análisis de causa. }\end{array}$} \\
\hline & & & $\begin{array}{l}\text { al producto } \\
\text { mayores a } 3 \mathrm{~mm}\end{array}$ & \\
\hline & Harin & & Presencia de & \\
\hline & Integrales & & partículas extrañas & \\
\hline & & & producto & \\
\hline & & & mayores a $6 \mathrm{~mm}$ & \\
\hline & & & & \\
\hline & & & & \\
\hline & & & & \\
\hline
\end{tabular}

Fuente. Elaborador por los autores

Se estableció en el Equipo HACCP el procedimiento de verificación para evitar peligros que atenten contra la inocuidad del producto, para ello se contará con el apoyo del personal del Departamento de Aseguramiento de Calidad con el siguiente procedimiento: Para Harinas, se tamizará el producto en el proceso de ensacado cada ocho horas por una malla de tres milímetros, donde debe haber una retención del cero por ciento. Con ello se asegura que el Punto Crítico de Control esté trabajando adecuadamente y se verifica la normalidad en el proceso garantizando la inocuidad, en donde, se obtuvo un $0 \%$ de retención.

Tabla V. Establecimiento de procedimientos de verificación

\begin{tabular}{|c|c|c|c|c|}
\hline PCC & Producto & Peligro & Límite crítico & Verificación \\
\hline \multirow[t]{2}{*}{$\begin{array}{l}\text { Tamizado de } \\
\text { control } \\
\text { turbotamizadora } \\
\text { MKZF }\end{array}$} & Harinas & $\begin{array}{l}\text { Partículas } \\
\text { extrañas al } \\
\text { producto } \\
\text { cuyo tamaño } \\
\text { pueda } \\
\text { afectar a la }\end{array}$ & $\begin{array}{lr}\text { Presencia } & \mathrm{de} \\
\text { partículas extrañas } \\
\text { al producto mayores } \\
\text { a } 3 \mathrm{~mm}\end{array}$ & $\begin{array}{l}\text { Tamizado de producto del } \\
\text { ensacado por malla de } 3 \mathrm{~mm} \text {. } \\
\text { Retención de } 0\end{array}$ \\
\hline & $\begin{array}{l}\text { Harinas } \\
\text { Integrales }\end{array}$ & $\begin{array}{l}\text { integridad o } \\
\text { la salud } \\
\text { humana }\end{array}$ & $\begin{array}{lr}\text { Presencia } & \mathrm{de} \\
\text { partículas extrañas } \\
\text { al producto mayores } \\
\text { a } 6 \mathrm{~mm}\end{array}$ & $\begin{array}{l}\text { Tamizado de producto del } \\
\text { ensacado por malla de } 6 \mathrm{~mm} \text {. } \\
\text { Retención de } 0 \text { \% } \\
\text { Cada lote de producción } \\
\text { Control de Calidad realizará la } \\
\text { verificación. }\end{array}$ \\
\hline
\end{tabular}

Fuente. Elaborador por los autores

Además, se estableció que se deben cumplir con los siguientes procesos de verificación:

Tabla VI. Procesos de Verificación en el mantenimiento del Sistema HACCP

\begin{tabular}{lrll}
\hline ACTIVIDAD & FRECUENCIA & RESPONSABLE \\
\hline Auditorías Internas & Anualmente & Equipo HACCP \\
\hline $\begin{array}{l}\text { Revisión de } \\
\text { desviaciones en } \\
\text { monitoreo de PCC }\end{array}$ & $\begin{array}{r}\text { las } \\
\text { el }\end{array}$ & Semestralmente & Equipo HACCP \\
\hline $\begin{array}{l}\text { Validación del Sistema } \\
\text { HACCP }\end{array}$ & $\begin{array}{l}\text { Cuando existan cambios en el proceso, reclamos } \\
\text { reglamentarios, nuevos peligros, cambios de } \\
\text { maquinaria, después de una falla en el sistema, etc. }\end{array}$ & \\
\hline
\end{tabular}

Fuente. Elaborador por los autores 


\section{V.CONCLUSIONES}

Se determinó que el Ecuador en su proceso de cambio de matriz productiva necesita incrementar el número de industrias alimenticias que posean sistemas de gestión de calidad e inocuidad, para ello sirve este trabajo como guía o pauta para la implementación del sistema de Evaluación basado en las HACCP.

Los responsables directos del cumplimiento de este sistema serán la gerencia General, gerencia Técnica; Comité de inocuidad, Jefes de Áreas. La validación del plan HACCP se la realizará anualmente por medio de auditorías internas o en caso de que exista un desvío considerable en el sistema, tales como: Cambios en el proceso, reclamos en el mercado, quejas de clientes, cambios reglamentarios, nuevos peligros. En caso de presentarse observaciones o hallazgos se realizará el respectivo plan de acción descrito en el formato HACCP R-02.

Se estableció que este análisis no puede ser aplicado a otra industria diferente al procesamiento de trigo ya que son procesos específicos y los peligros hacia la inocuidad varían de industria a industria. Se concluyó que el acceso a la información relacionada con procesamiento y análisis de cereales es limitado, por tal razón la industria dedicada a este rubro busca alternativas para su obtención en otros países o instituciones educativas especializadas.

Fomentar este tipo de desarrollos para otras industrias alimenticias ya que genera confianza y fidelidad en el consumidor final. Promover en nuestro país el acceso a la información relacionada a molinería de trigo y cereales, buscando apertura en la industria local, reforzando bases de datos o textos académicos, realizando seminarios, siendo generadores de investigación y ciencia para poder aplicarla en el negocio nacional y ser más competitivos globalmente.

\section{REFERENCIAS}

[1]D. Palacios and P. Reyes, "Cambio de la matriz productiva del Ecuador y su efecto en el comercio exterior," Dominio las Ciencias, vol. 2, pp. 418-431, 2016. [2]FDA, "National Advisory Committee on microbiological criteria for foods," United States Food Drug
Adm., 2015.

[3]OIRSA, "Manual de análisis de peligros y puntos críticos de control - HACCP." OIRSA, p. 32, 2016.

[4]Organización Panamericana de la Salud, "Sistema HACCP." 2015.

[5]J. Anaya and S. Polanco, Innovación y Mejora de Procesos Logísticos, Segunda ed. España: ESIC, 2007.

[6]FAO, "Manual sobre la aplicación del Análisis de Peligros y de Puntos Críticos de control (APPCC) en la prevención y control de micotoxinas." FAO, p. 136, 2015.

[7]Instituto Nacional de Normalización, Sistema de análisis de peligros y de puntos críticos de control (HACCP), directrices para su ejecución. Chile, 2011, p. 27.

[8]M. Jaramillo, "Desarrollo de un Sistema de Análisis de Peligros y Puntos Críticos de control ( HACCP ) para garantizar la inocuidad alimentaria en una Industria Molinera de trigo," Pontificia Universidad Católica del Ecuador- Sede Ambat, 2015.

[9]G. y P. Ministerio de Agricultura, "Sistemas de gestión de la calidad en el sector agroalimentario." Ministerio de Agricultura, Ganadería y Pesca - Argentina, Argentina, pp. 87-93, 2016.

[10]M. Alvarez, "Procedimientos y programas de seguridad alimentaria: HACCP, GMP Control de calidad del agua, zonificación y sanitización alimentaria en la industria de gelatinas," Universidad San Francisco de Quito, 2006.

[11]L. Bou, N. Ascanio, and P. Hernández, "Diseño de un plan de análisis de peligros y puntos críticos de control (HACCP) para el aseguramiento de la inocuidad de la mortadela elaborada por una empresa de productos cárnicos," Arch. Latinoam. Nutr., vol. 54, no. 1, 2004. [12]A. Paccha, "Implementación de nuevos procesos de mantenimiento de maquinarias de producción para optimizar la rentabilidad del en el negocio de la harina de trigo," Escuela Superior Politécnica del Litoral, 2011.

[13]Codex, "Norma del Codex para el trigo y el trigo duro." Codex Standard, 1995.

[14]R. Hernández - Sampieri, C. Fernández, and P. Baptista, Metodología de la investgación, Sexta edic. México D.F: McGRW HILL, 2014. 